\title{
Exciton and charge-transfer dynamics in polymer semiconductors
}

\author{
Eric R. Bittner and John Glen S. Ramon \\ Department of Chemistry and Center for Materials Chemisty, University of Houston, Houston, TX
}

(Dated: April 25, 2022)

\begin{abstract}
Organic semiconducting polymers are currently of broad interest as potential low-cost materials for photovoltaic and light-emitting display applications. I will give an overview of our work in developing a consistent quantum dynamical picture of the excited state dynamics underlying the photo-physics. We will also focus upon the quantum relaxation and reogranization dynamics that occur upon photoexcitation of a couple of type II donor-acceptor polymer heterojunction systems. Our results stress the significance of vibrational relaxation in the state-to- state relaxation and the impact of curve crossing between charge- transfer and excitonic states. Furthermore, while a tightly bound charge-transfer state (exciplex) remain the lowest excited state, we show that the regeneration of the optically active lowest excitonic state in TFB:F8BT is possible via the existence of a steadystate involving the bulk charge-transfer state. Finally, we will discuss ramifications of these results to recent experimental studied and the fabrication of efficient polymer LED and photovoltaics.
\end{abstract}

\section{INTRODUCTION}

Organic semiconducting materials have attracted the attention of the electronic industry. While the low charge mobility compared to crystalline silicon and various other characteristics limit the range of potential application for organics, a wide range of properties such as solubility in organic solvents and the color of light emission can be finely tuned using ordinary chemical synthesis. Molecules and their arrangement can be easily engineered to fit a specific requirement. Moreover, polymer coatings can be easily applied to a wide range of surfaces and media, including mechanically flexible ones, to fabricate large scale displays or electronic sensors with complex shapes. Organic electronics complement and extend the range of traditional silicon based electronics. The chemical structures of various common semiconducting polymer chains are given in Fig 1 In practice, PPV is often functionalized with alkyl side chains in order to tune its electronic properties and increase its solubility in organic solvents.

Organic LED devices are typically layered structures with luminescent media sandwiched between cathode and anode materials which are selected such that their Fermi energies roughly match the conduction and valence bands of the luminescent material. Often the semiconducting media itself consists of a hole transport layer and an electron transport layer engineered to facilitate the rapid diffusion of the injected carriers away from their image charges on the cathode or anode. These carriers are best described as polarons since electron-phonon coupling produces significant lattice reorganization about the carriers. Finally, a third, luminescent layer can be sandwiched between the transport layers. In this layer, the electron and hole polarons interact and combine to produce excitons. The individual spins of the electrons and holes are uncorrelated and only singlet excitons are radiatively coupled the ground electronic state. In the absence of singlettriplet coupling, this places a theoretical upper-limit or 1:4 or $25 \%$ on the overall efficiency of a LED device and it has been long debated whether or not the efficiency of organic LED devices is in fact limited by this theoretical

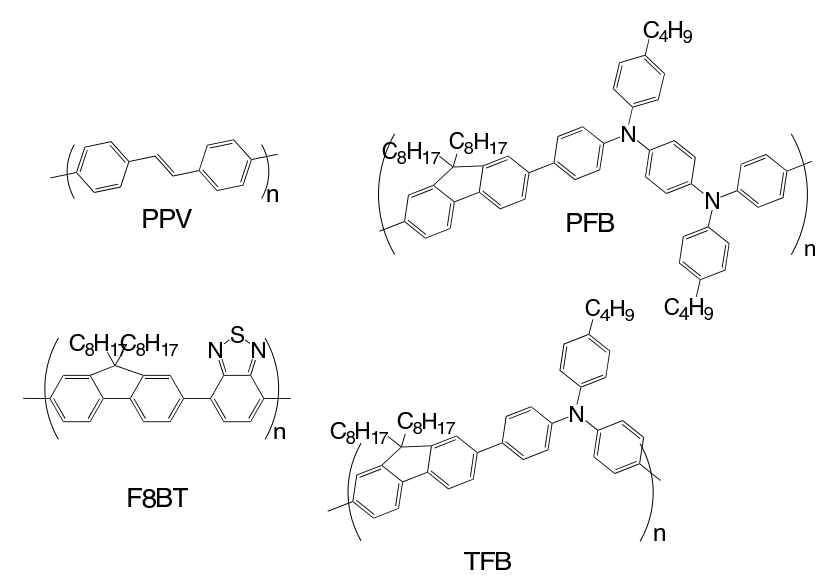

FIG. 1: Structures and common short-hand names of various conjugated poly-phenylene derived semiconducting polymers that are of interest for fabricating luminescent devices.

\section{upper-limit.}

The electronic properties of these materials are derived from the delocalized $\pi$ orbitals found in conjugated polymers. The $\pi$ electron system is primarily an intramolecular network extending along the polymer chain. For a linear chain, the valance and conduction $\pi$ and $\pi^{*}$ bands are typically $1-3 \mathrm{eV}$ wide compared to the intermolecular bandwidth (due to $\pi$-stacking) of about $0.1 \mathrm{eV}$ for well ordered materials. Thus, intrachain charge transport is extremely efficient; however, interchain transport typically limits the charge mobility for the usual size range of devices. The polymer backbone is held together through a $\sigma$ bonding network. These bonds are considerably stronger than the $\pi$ bonds and keep the molecule intact even following photoexcitation. Hence, we can consider the electronic dynamics as taking place within the $\pi$ band and treat the localized $\sigma$ bonds as skeletal framework.

Since the dielectric constant of organic semiconductors is relatively low, screening between charges is relatively week. At a given radius, $r_{c}$, thermal fluctuation will be 
insufficient to break apart an electron/hole pair,

$$
k T=\frac{e^{2}}{\epsilon r_{c}}
$$

at $300 \mathrm{~K}$, this radius is approximately $20 \mathrm{~nm}$, which is on the order of a few molecular lengths. If we consider the electron/hole pair to be a hydrogenic-type system with effective masses equal to the free electron mass and dielectric constant of 3 , the resulting binding energy is about $0.75 \mathrm{eV}$ with an effective Bohr radius of $0.3 \mathrm{~nm}$, which effectively confines the exciton to a single molecular unit. Finally, if we consider the electron/hole pair to be pair of bound Fermions, exchange energy resulting from the anti-symetrization of the electron/hole wave function splits the spin-singlet and spin-triplet excitons by about $0.5-0.7 \mathrm{eV}$ with the spin-triplet lying lower in energy than the singlet. While both species are relatively localized, singlets typically span about $10 \mathrm{~nm}$ in well ordered materials while triplets are much more localized. In absence of spin-orbit coupling, emission from the triplet states is forbidden. Hence, triplet formation in electron/hole capture can dramatically limit the efficiency of a light-emitting diode device, although strong theoretical and experimental evidence indicates that singlet formation can be enhanced in long-chain polymers.

Experiments by various groups suggest that in longchain conjugated polymer systems, the singlet exciton population can be greatly enhanced and that efficiencies as high as 60 to $80 \%$ can be easily achieved in PPV type systems. 1] On the other hand, in small oligomers, the theoretical upper limit appears to hold true. These initial experiments were then followed by a remarkable set of observations by Wohlgenannt and Vardeny [2] that indicate that the singlet to triplet ratio, $r>1$ for wide range of conjugated polymer systems and that $r$ scales universally with the polaron energy-which itself scales inversely with the persistence length of the $\pi$-conjugation,

$$
r \propto 1 / n .
$$

The electro-luminescent efficiency, $\phi$, is proportional to the actual singlet population and is related to $r$ via

$$
\phi=r /(r+3) .
$$

Various mechanisms favoring the formation of singlets have been proposed for both interchain and intrachain e-h collisions. Using Fermi's golden rule, Shuai, Bredas et al. [3, [4, [5] indicate that the $S$ cross section for interchain recombination can be higher than the triplet one due to bond-charge correlations. Wohlgenannt et al. [6] employ a similar model of two parallel polyene chains. Both of these works neglect vibronic and relaxation effects. In simulating the intrachain collision of opposite polarons, Kobrak and Bittner [7, 8, 9] show that formation of singlets are enhanced by the near-resonance with the free e-h pair. The result reflects the fact that spinexchange renders the triplet more tightly bound than the singlet and hence more electronic energy must be dissipated by the phonons in the formation of the former. The energy-conservation constraints in spin-dependent e-h recombination have been analyzed by Burin and Ratner [10] in an essential-state model. The authors point out that nonradiative processes (internal conversion, intersystem crossing) must entail $\mathrm{C}=\mathrm{C}$ stretching vibrons since these modes couple most strongly to $\pi \rightarrow \pi^{*}$ excitations. Tandon et al. suggest that irrespective of the recombination process, interchain or intrachain, the $d i$ rect transition to form singlets should always be easier than triplets due to its smaller binding energy relative to the triplet. 11]. A comprehensive review of detailing the experiments and theory of this effect was presented by Wohlgenannt and Vardeny, 12]. By and large, recent theoretical models point towards the role of multi-phonon relaxation and the scaling of the singlet/triplet splitting with chain length as dominant factors in determining this enhancement. 13, 14, 15, 16]

If we assume that the electron/hole capture proceeds via a series of microstates one can show that the ratio of the singlet to triplet capture cross-sections, $r$ scale with the ratio of the exciton binding energies 17 .

$$
r \propto \frac{\epsilon_{B}^{T}}{\epsilon_{B}^{S}} .
$$

If we take the singlet-triplet energy difference to be equal to twice the electron-hole exchange energy, $\Delta E_{S T}=2 K$, and expand $K$ in terms of the inverse conjugation length,

$$
K=K_{\infty}+K^{(1)} / n+\cdots
$$

where $2 K_{\infty}$ is the singlet-triplet splitting of an infinitely long polymer chain, one obtains

$$
r \propto 1+n K_{\infty} / \epsilon_{B}^{S}+\cdots
$$

Since both $K_{\infty}>0, r>1$. Moreover, if we take $\epsilon_{B}^{S} \propto 1 / n$, we obtain a simple and universal scaling law for the singlet-triplet capture ratio, $r \propto n$. This "universal" scaling law for $r$ was reported by Wohlgennant and Vardeny [2]. What is even more surprising, is that the the same scaling law (i.e. slope and intercept for $r=a n+c$ ) describes nearly all organic conjugated polymer systems. Hence, it appears that a common set of electronic interaction parameters is transferable between a wide range of organic conjugated polymer systems.

Another general consequence of localized electronic states in molecular semiconductors is their effect on the molecule itself. Promoting an electron from a $\pi$ bonding orbital to a $\pi^{*}$ anti-bonding orbital decreases the bond order over several carbon-carbon bonds. This leads to a significant rearrangement of the bond-lengths to accommodate the changes in the electronic structure. By in large, for polymers containing phenyl rings, it is the $\mathrm{C}=\mathrm{C}$ bond stretching modes and much lower frequency phenylene torsional modes that play significant roles in the lattice reorganization following optical excitation. This is 


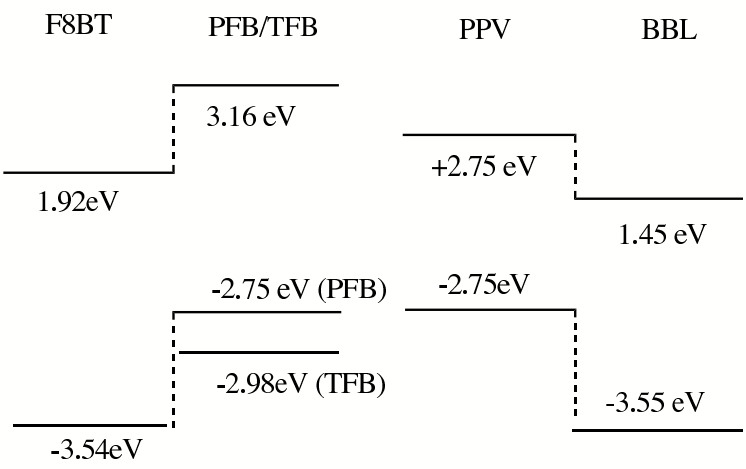

FIG. 2: Relative placement of the HOMO and LUMO levels for various conjugated polymers.

evidenced in the strong vibronic features observed in the absorption and emission spectra of these materials.

Finally, one can fabricate devices using blends of semiconducting polymers which phase segregate. For example, the phenylene backbone in F8BT is very planar molecule facilitating very delocalized $\pi$-states. On the other hand, TFB and PFB are very globular polymers due to the tri-amide group in the chain. Consequently, phase segregation occurs due to more favorable $\pi$-stacking interactions between F8BT chains than between F8BT and TFB or PFB. Moreover, the electronic states in TFB and PFB are punctuated by the tri-amides. This difference in electronic states results in a band offset between the two semiconducting phases. When we place the materials in contact with each other, a $p$ - $n$ heterojunction forms.

One can think of the HOMO and LUMO energy levels of a given polymer as corresponding to the top and bottom of the valance and conduction bands respectively. For the polymers under consideration herein, the relative band edges are shown in Fig. 22 In Type II heterojunction materials, the energy bands of the two materials are off set by $\Delta E$. If the exciton binding energy $\varepsilon_{B}>\Delta E$, excitonic states will the lowest lying excited state species, resulting in a luminescent material with the majority of the photons originating from the side with the lowest optical gap. Since the majority of the charge carriers are consumed by photon production, very little photocurrent will be observed.

On the other hand, charge transfer states across the interface will be energetically favored if $\varepsilon_{B}<\Delta E$. Here, any exciton formed will rapidly decay in to a chargeseparated state with the electron and hole localizing on either side of the junction. This will result in very little luminescence but high photocurrent. Consequently, heterojunctions of PPV and BBL which have a large band off-set relative to the exciton binding energy are excellent candidate materials for organic polymer solar cells.

Heterojunctions composed of TFB:F8BT and PFB:F8BT lie much closer to the exciton stabiliza- tion threshold as seen by comparing the relative band off-sets in Fig 2 Notice that the off-set for TFB:F8BT is only slightly larger than $0.5 \mathrm{eV}$, which is approximately the exciton binding energy where as in PFB:F8BT the off-set is $>0.5 \mathrm{eV}$. Since such blends lie close to the stabilization threshold, they are excellent candidates for studying the relation between the energetics and the kinetics of exciton fission.

In this paper we will present an overview of our recent theoretical work aimed at understanding and modeling the state-to-state photophysical pathways in blended heterojunction materials. 18, 19]

\section{TWO-BAND CONFIGURATION INTERACTION MODEL}

Our basic description is derived starting from a model for the on-chain electronic excitations of a single conjugated polymer chain. 16, 20, 21] This model accounts for the coupling of excitations within the $\pi$-orbitals of a conjugated polymer to the lattice phonons using localized valence and conduction band Wannier functions $(|\bar{h}\rangle$ and $|p\rangle)$ to describe the $\pi$ orbitals and two optical phonon branches to describe the bond stretches and torsions of the the polymer skeleton.

$$
\begin{aligned}
H & =\sum_{\mathbf{m n}}\left(F_{\mathbf{m n}}^{\circ}+V_{\mathbf{m n}}\right) A_{\mathbf{m}}^{\dagger} A_{\mathbf{n}} \\
& +\sum_{\mathbf{n} \mathbf{m} i \mu}\left(\frac{\partial F_{\mathbf{n} \mathbf{m}}^{\circ}}{\partial q_{i \mu}}\right) A_{\mathbf{n}}^{\dagger} A_{\mathbf{m}} q_{i \mu} \\
& +\sum_{i \mu} \omega_{\mu}^{2}\left(q_{i \mu}^{2}+\lambda_{\mu} q_{i \mu} q_{i+1, \mu}\right)+p_{i \mu}^{2}
\end{aligned}
$$

where $A_{\mathbf{n}}^{\dagger}$ and $A_{\mathbf{n}}$ are Fermion operators that act upon the ground electronic state $|0\rangle$ to create and destroy electron/hole configurations $|n\rangle=|\bar{h} p\rangle$ with positive hole in the valence band Wannier function localized at $h$ and an electron in the conduction band Wannier function $p$. Finally, $q_{i \mu}$ and $p_{i \mu}$ correspond to lattice distortions and momentum components in the $i$-th site and $\mu$-th optical phonon branch.

Wannier functions are essentially spatially localized basis functions that can be derived from the bandstructure of an extended system. Quantities such as the exchange interaction and Coulomb interaction can be easily computed within the atomic orbital basis; however, there are many known difficulties in computing these within the crystal momentum representation. Because of this, is is desirable to develop a set of orthonormal spatially localized functions that can be characterized by a band index and a lattice site vector, $R_{\mu}$. These are the Wannier functions, which we shall denote by $a_{n}\left(r-R_{\mu}\right)$ and define in terms of the Bloch functions

$$
a_{n}\left(r-R_{\mu}\right)=\frac{\Omega^{1 / 2}}{(2 \pi)^{d / 2}} \int e^{-i k R_{\mu}} \psi_{n k}(r) d k .
$$


The integral is over the Brillouin zone with volume $V=(2 \pi)^{d} / \Omega$ and $\Omega$ is the volume of the unit cell (with $d$ dimensions). A given Wannier function is defined for each band and for each unit cell. If the unit cell happens to contain multiple atoms, the Wannier function may be delocalized over multiple atoms. The functions are orthogonal and complete.
The Wannier functions are not energy eigenfunctions of the Hamiltonian. They are, however, linear combinations of the Bloch functions with different wave vectors and therefore different energies. For a perfect crystal, the matrix elements of $H$ in terms of the Wannier functions are given by

$$
\begin{aligned}
\int a_{l}^{*}\left(r-R_{\nu}\right) H_{o} a_{n}\left(r-R_{\mu}\right) d r & =\frac{\Omega}{(2 \pi)^{d}} \int e^{i\left(q R_{\nu}-k R_{\mu}\right)} \psi_{l k}(r) H_{o} \psi_{n k}(r) d r d q d k \\
& =\mathcal{E}_{n}\left(R_{\nu}-R_{\mu}\right) \delta_{n l}
\end{aligned}
$$

where

$$
\mathcal{E}_{n}\left(R_{\nu}-R_{\mu}\right)=\frac{\Omega}{(2 \pi)^{d}} \int e^{i k\left(R_{\nu}-R_{\mu}\right)} E_{n}(k) d k .
$$

Consequently, the Hamiltonian matrix elements in the Wannier representation are related to the Fourier components of the band structure, $E_{n}(k)$. Therefore, given a band structure, we can derive the Wannier functions and the single particle matrix elements, $F_{\mathbf{m n}}^{\circ}$.

The single-particle terms, $F_{\mathbf{m n}}^{\circ}$, are derived at the ground-state equilibrium configuration, $q_{\mu}=0$, from the Fourier components $f_{r}$ and $\bar{f}_{r}$ of the band energies in pseudomomentum space.

$$
\begin{aligned}
F_{\mathbf{m n}} & =\delta_{\overline{m n}}\langle m|f| n\rangle-\delta_{m n}\langle\bar{m}|\bar{f}| \bar{n}\rangle \\
& =\delta_{\overline{m n}} f_{m-n}-\delta_{m n} \bar{f}_{\bar{m}-\bar{n}}
\end{aligned}
$$

Here, $f_{m n}$ and $\bar{f}_{m n}$ are the localized energy levels and transfer integrals for conduction-band electrons and valence-band holes. At the ground-state equilibrium geometry, $q_{\mu}=0$, these terms can be computed as Fourier components of the one-particle energies in the Brillouin zone. For example, for the conduction band:

$$
f_{m n}=f_{m-n}=\frac{1}{B_{z}} \int_{B_{z}} \varepsilon_{k} e^{i k(m-n)} d k
$$

where $k$ is the pseudomomentum for a 1-dimensional lattice with unit period. For the case of cosine-shaped bands,

$$
\epsilon(k)=f_{o}+2 f_{1} \cos (k)
$$

the site-energies are given by the center $f_{o}$ and the transfer integral between adjacent Wannier functions is given by $f_{1}$. The band-structure and corresponding Wannier functions for the valence and conduction bands for PPV are shown in Fig. 3] 15, 20, 21] For the intrachain terms, we use the hopping terms and site energies derived for isolated polymer chains of a given species, $t_{i, \|}$, where our notation denotes the parallel hopping term for the $i$ th chain $(i=1,2)$. For PPV and similar conjugated polymer species, these are approximately $0.5 \mathrm{eV}$ for both valence and conduction $\pi$ bands.

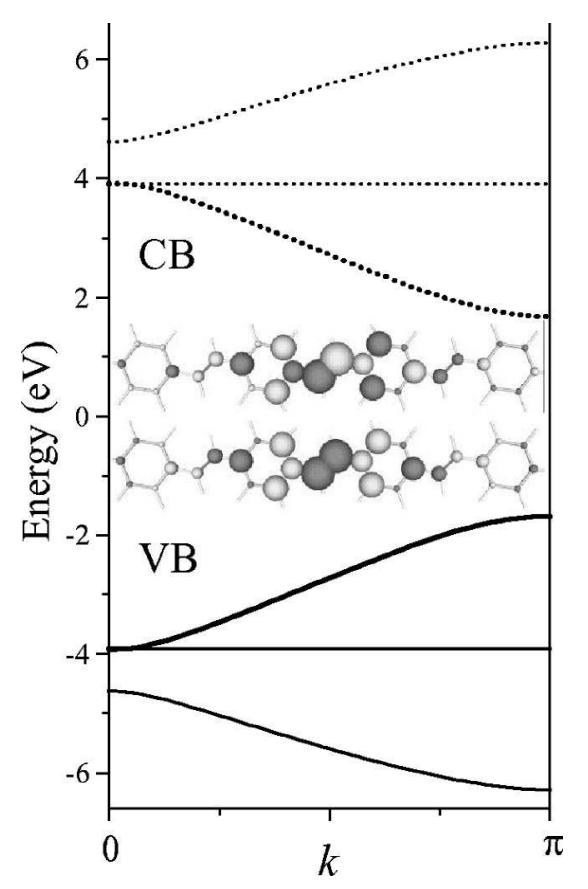

FIG. 3: Computed band-structure and vinylene-centered Wannier functions for PPV.

The two-particle interactions are spin dependent with

$$
\begin{aligned}
& V_{m n}^{T}=-\langle m \bar{n} \| n \bar{m}\rangle \\
V_{m n}^{S}= & V_{m n}^{T}+2\langle m \bar{n} \| \bar{m} n\rangle .
\end{aligned}
$$


TABLE I: Electron/hole integrals for poly-phenylene-type polymer chains.

\begin{tabular}{c|c|c}
\hline \hline Term & Functional form & Parameters \\
\hline Direct Coloumb & $J(r)=J_{o} /\left(1+r / r_{o}\right)$ & $\begin{array}{c}J_{o}=3.092 \mathrm{eV} \\
r_{o}=0.6840 a\end{array}$ \\
\hline Exchange & $K(r)=K_{o} e^{-r / r_{o}}$ & $\begin{array}{c}K_{o}=1.0573 \mathrm{eV} \\
r_{o}=0.4743 a\end{array}$ \\
\hline Dipole-Dipole & $D(r)=D_{o}\left(r / r_{o}\right)^{-3}$ & $\begin{array}{c}D_{o}=-0.03209 \mathrm{eV} \\
r_{o}=1.0 a\end{array}$ \\
\hline \hline
\end{tabular}

Note: $a=$ unit lattice spacing.

for triplet and singlet combinations respectively with

$$
\langle m \bar{n} \| i \bar{j}\rangle=\int d 1 \int d 2 \phi_{m}^{*}(1) \phi_{\bar{n}}^{*}(2) v(12) \phi_{i}(1) \phi_{\bar{j}}(2)
$$

With the exception of geminate WFs, orbital overlap is small such that the two-body interactions are limited to Coulomb, $J(r)$ and exchange integrals, $K(r)$ reflecting e-h attraction and spin-exchange coupling nongeminate configurations. and transition dipole-dipole integrals $D(r)$ coupling only geminate singlet electron-hole pairs. Table \gives a listing of the electron-hole integrals and their parameters we have determined for PPV and similar poly-phenylene based conjugated chains. We have found that these are quite transferable amongst this class of conjugated polymers and allow us to focus upon modeling similar poly-phenylene chains through variation of the Wannier function band-centers (i.e. site-energies) and band-widths (i.e. intrachain hopping integrals).

Since we will be dealing with inter-chain couplings, we make the following set of assumptions. First, the single-particle coupling between chains is expected to be small compared to the intramolecular coupling. For this, we assume that the perpendicular hopping integral $t_{\perp}=0.01 \mathrm{eV}$. This is consistent with LDA calculations performed by Vogl and Campbell and with the $t_{\perp} \approx 0.15 f_{1}$ estimate used in an earlier study of interchain excitons by $\mathrm{Yu}$ et al. 22, 23] Furthermore, we assume that the $J(r), K(r)$, and $D(r)$ two-particle interactions depend only upon the linear distance between two sites, as in the intrachain case. Since these are expected to be weak given that the interchain separation, $d$, is taken to be some what greater than the inter-monomer separation.

An important component in our model is the coupling between the electronic and nuclear degrees of freedom. These we introduce via a linear coupling term:

$$
\left(\frac{\partial f_{m n}}{\partial q_{\mu}}\right)_{\circ}=\frac{S}{2}\left(2 \hbar \omega^{3}\right)^{1 / 2}\left(\delta_{m \mu}+\delta_{n \mu}\right)
$$

where $S$ is the Huang-Rhys factor which can be obtained from vibronic features in the experimental photoemission spectra. For the case of conjugated polymers such as PPV and similar poly-phenylene species, the emission spectra largely consists of a series of well-resolved vibronic features corresponding to the $\mathrm{C}=\mathrm{C}$ stretching modes in the phenylene rings with typical Huang Rhys factors of 0.4. In addition, low frequency vibrational modes (due to torsions) contribute a broad featureless background. Consequently. we include two intramolecular optical phonon branches which correspond roughly to the high-frequency $\mathrm{C}=\mathrm{C}$ bond stretching modes within a given repeat unit and a second low-frequency mode, which in the case of PPV are taken to represent the phenylene torsional modes. The electron-phonon couplings are assumed to be transferable between the various chemical species. Since the modes are assumed to be intramolecular, we do not include interchain couplings in the phonon Hessian matrix.

Upon transforming $H$ into the diabatic representation by diagonalizing the electronic terms at $q_{i \mu}=0$, we obtain a series of vertical excited states $\left|a_{\circ}\right\rangle$ with energies, $\varepsilon_{a}^{\circ}$ and normal modes, $Q_{\xi}$ with frequencies, $\omega_{\xi}$. (We will assume that the sum over $\xi$ spans all phonon branches).

$$
\begin{aligned}
H & =\sum_{a} \varepsilon_{a}^{\circ}\left|a_{\circ}\right\rangle\left\langle a_{\circ}\right|+\sum_{a b \xi} g_{a b \xi}^{\circ} q_{\xi}\left(\left|a_{\circ}\right\rangle\left\langle b_{\circ}|+| b_{\circ}\right\rangle\left\langle a_{\circ}\right|\right) \\
& +\frac{1}{2} \sum_{\xi}\left(\omega_{\xi}^{2} Q_{\xi}^{2}+P_{\xi}^{2}\right) .
\end{aligned}
$$

The adiabatic or relaxed states can be determined then by iteratively minimizing $\varepsilon_{a}\left(Q_{\xi}\right)=\langle a|H| a\rangle$ according to the self-consistent equations

$$
\frac{d \varepsilon_{a}\left(Q_{\xi}\right)}{d Q_{\xi}}=g_{a a \xi}+\omega_{\xi}^{2} Q_{\xi}=0
$$

Thus, each diabatic potential surface for the nuclear lattice motion is given by

$$
\varepsilon_{a}\left(Q_{\xi}\right)=\varepsilon_{a}+\frac{1}{2} \sum_{\xi} \omega_{\xi}^{2}\left(Q_{\xi}-Q_{\xi}^{(a)}\right)^{2} .
$$

These are shown schematically as $S_{a}$ and $S_{b}$ in Fig. 4 with $Q_{\xi}$ being a collective normal mode coordinate. On can also view this figure as a slice through an $N$-dimensional coordinate space along normal coordinate $Q_{\xi}$ In this figure, $\epsilon_{a}^{\circ}$ and $\epsilon_{b}^{\circ}$ are the vertical energies taken at the ground-state equilibrium geometry $Q_{\xi}=0$. The adiabatic energies, taken at the equilibrium geometry of each excited state are denoted as $\epsilon_{a}$ and $\epsilon_{b}$. While our model accounts for the distortions in the lattice due to electron/phonon coupling, we do not account for any adiabatic change in the phonon force constants within the excited states. Lastly, the electronic coupling between diabatic curves is given by $g_{a b}$ which we compute at the ground-state geometry $\left(g_{a b}^{\circ}\right)$. We assume that both the diagonal $g_{a a}^{\circ}$ and off-diagonal $g_{a b}^{\circ}$ terms can be derived from the spectroscopic Huang-Rhys parameters as in Eq. 9

The advantages of our approach is that it allows us to easily consider the singly excited states of relatively large 


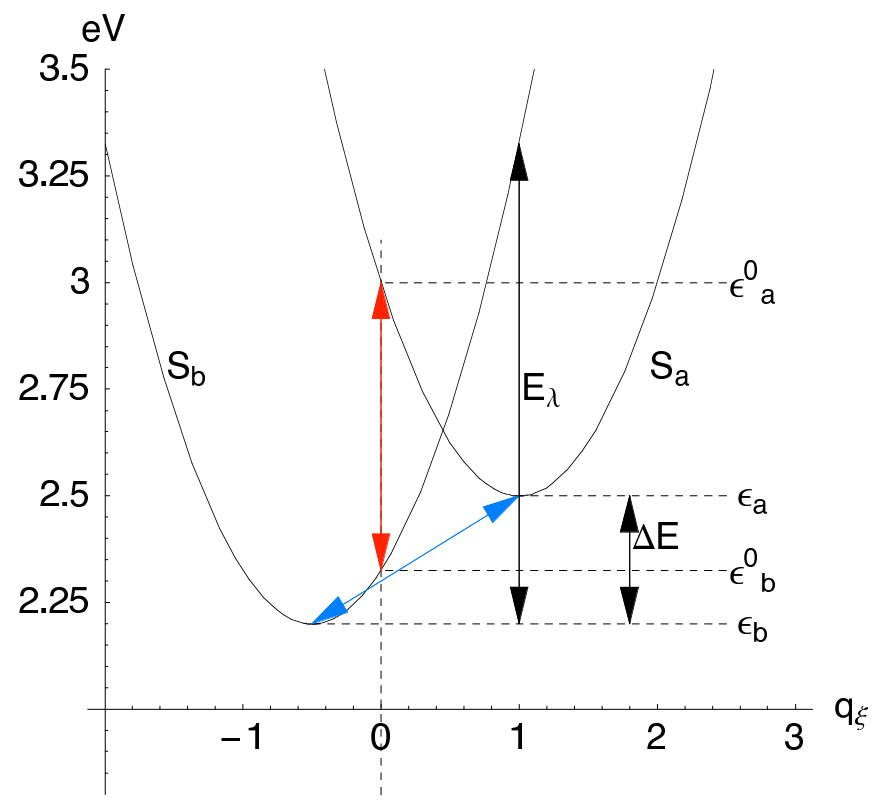

FIG. 4: Schematic representation of excited state Diabatic potentials obtained within our approach. The ground state configuration is taken as $q_{\xi}=0$ with vertical excitation energies at $\epsilon_{a}^{\circ}$ and $\epsilon_{b}^{\circ}$ and adiabatic (minimum) energies at $\epsilon_{a}$ and $\epsilon_{b}$.

conjugated polymer systems. Our model is built from both $a b$ initio and experimental considerations and can in fact reproduce most of the salient features of the vibronic absorption and emission spectra for these systems. The model is limited in that we cannot include specific chemical configurational information about the polymers other than their conjugation length and gross topology. For isolated single chains, the model is rigorous. For multiple chains, our interchain parameterization does not stand on such firm ground since technically the Wannier functions are derived from a quasi-one-dimensional band structure. Nonetheless, our model and results provide a starting point for predicting and interpreting the complex photophysical processes within these systems. We next move on to describing the state-to-state interconversion proceses that occur following both photo and electro-excitation.

\section{STATE TO STATE RELAXATION DYNAMICS}

The electronic levels in our model are coupled to the lattice phonons as well as the radiation field. Consequently, relaxation from a given electronic state can occur via state to state interconversion via phonon excitation or absorption or fluorescent decay to the $S_{o}$ ground state. For triplet excitations, only phonon transitions are allowed. For the singlets, fluorescence occurs primarily from the lowest $S_{n}$ state independent of how the excitation was prepared. This certainly holds true for con- jugated polymers in which both electroluminescence and photoluminescence originates from the same $S_{1}=S_{x t}$ state. This implies that internal conversion dynamics are fast relative to the fluorescence lifetime.

Coupling the electronic relaxation dynamics to the vibrational dynamics is a formidable task. An exact quantum mechanical description of this is currently well beyond the state of the art of current computational methods. One can, however, compute the state-to-state rate constants using Fermi's golden rule and arrive at a reasonable picture.

If we assume that the vibrational bath described by $H_{p h}$ remains at its ground-state geometry, then the stateto-state transition rates are easily given by Fermi's golden rule:

$k_{a b}^{\circ}=\pi \sum_{\xi} \frac{g_{a b}^{2}}{\hbar \omega_{\xi}}\left(1+n\left(\omega_{a b}\right)\right)\left(\Gamma\left(\omega_{\xi}-\omega_{a b}\right)-\Gamma\left(\omega_{\xi}+\omega_{a b}\right)\right)$

where $n(\omega)$ is the Bose-Einstein population for the phonons, $\Gamma$ is a Lorentzian broadening in which the width is inversely proportional to the phonon lifetime used to smooth the otherwise discrete phonon spectrum, $\omega_{a b}=\left(\epsilon_{a}^{o}-\epsilon_{b}^{o}\right) / \hbar$. In order for a transition to occur, there must be a phonon of commensurate energy to accommodate the energy transfer. The coupling term, $g_{a b \xi}$, is the diabatic coupling in the diabatic Hamiltonian given in Eq. [10.

This static model is fine so long as either the nuclear relaxation has little effect on the state to state rate constant or if the electronic transitions occur on a time-scale which is short compared to the nuclear motion. However, if lattice reorganization does play a significant role, then we need to consider the explicit nuclear dynamics when computing the state-to-state rates. If we assume that vibrational relaxation within a given diabatic state is rapid compared to the inter-state transition rate, we can consider the transitions as occurring between displaced harmonic wells

$$
k_{a b}=\frac{2 \pi}{\hbar}\left|V_{a b}\right|^{2} \mathcal{F}
$$

where $V_{a b}$ is the coupling between electronic states $a$ and $b$ and

$$
\begin{aligned}
\mathcal{F}\left(E_{a b}\right) & =\sum_{\nu_{a}} \sum_{\nu_{b}} P_{t h}\left(\varepsilon_{a}\left(\nu_{a}\right)\right)\left|\left\langle\nu_{a} \mid \nu_{b}\right\rangle\right|^{2} \\
& \times \delta\left(\epsilon_{a}\left(\nu_{a}\right)-\epsilon\left(\nu_{b}\right)+\Delta E_{a b}\right)
\end{aligned}
$$

is the thermally averaged Franck-Condon weighted density of nuclear vibrational states. Here, $\nu_{a}$ and $\nu_{b}$ denote the vibronic states, $P_{t h}$ is the Boltzmann distribution over the initial states, $\epsilon_{a}\left(\nu_{a}\right)$ and $\epsilon\left(\nu_{b}\right)$ are the corresponding enregies, and $\Delta E_{a b}$ is the electronic energy gap between $a$ and $b$. In the classical limit, $\mathcal{F}$ becomes

$$
\mathcal{F}\left(E_{a b}\right)=\frac{1}{\sqrt{4 \pi E_{\lambda} k_{B} T}} \exp \left(-\frac{\left(E_{\lambda}+\Delta E_{a b}\right)^{2}}{4 E_{\lambda} k_{B} T}\right)
$$


where $E_{\lambda}$ is the reorganization energy as sketched in Fig. 4

Each of these terms can be easily computed from the diabatic Hamiltonian in Eq. 10. The diabatic coupling matrix element between the adiabatically relaxed excited states, $\left|V_{a b}\right|^{2}$, requires some care since we are considering transitions between eigenstates of different Hamitonians (corresponding to different nuclear geometries). Since the vertical $Q_{\xi}=0$ states provide a common basis, $\left|a^{\circ}\right\rangle$, we can write

$$
V_{a b}=\sum_{a^{\circ} b^{\circ}}\left\langle a \mid a^{\circ}\right\rangle g_{a b}^{\circ}\left\langle b^{\circ} \mid b\right\rangle
$$

where $g_{a b}^{\circ}$ is the diabatic matrix element computed at the equilibrium geometry of the ground-state.

Once we have the rate constants computed, it is a simple matter to integrate the Pauli master equation for the state populations.

$$
\dot{P}_{a}(t)=\sum_{b}\left(k_{b a} P_{b}-k_{a b} P_{a}\right)-k_{a}^{r a d} P_{a}
$$

where $k_{a}^{\text {rad }}$ is the radiative decay rate of state $a$.

$$
k_{a}^{r a d}=\frac{\left|\mu_{a 0}\right|^{2}}{6 \epsilon_{o} \hbar^{2}}\left(1+n\left(\omega_{a 0}\right)\right) \frac{\hbar \omega_{a 0}^{3}}{2 \pi c^{3}}
$$

where $\mu_{a 0}$ are the transition dipoles of the excited singlets. These we can compute directly from the Wannier functions or empirically from the photoluminescence decay rates for a given system. Photon mediated transitions between excited states are highly unlikely due to the $\omega_{a b}^{3}$ density factor of the optical field. In essence, so long as the non-equilibrium vibrational dynamics is not a decisive factor, we can use these equations to trace the relaxation of an electronic photo- or charge-transfer excitation from its creation to its decay including photon outflow measured as luminescence.

\section{EXCITON REGENERATION DYNAMICS}

Donor-acceptor heterojunctions composed of blends of TFB with F8BT and PFB with F8BT phase segregate to form domains of more or less pure donor and pure acceptor. Even though the polymers appear to be chemically quite similar, the presence of the tri-phenyl amine groups in TFB and PFB cause the polymer chain to be folded up much like a carpenter's rule. F8BT, on the other hand, is very rod-like with a radius of gyration being more or less equivalent to the length of a give oligomer. Molecular dynamics simulations of these materials by our group indicate that segregation occurs because of this difference in morphology and that the interface between the domains is characterized by regions of locally ordered $\pi$ stacking when F8BT rod-like chains come into contact with more globular PFB or TFB chains.

As discussed earlier, TFB:F8BT and PFB:F8BT sit on either side of the exciton destabalization threshold.
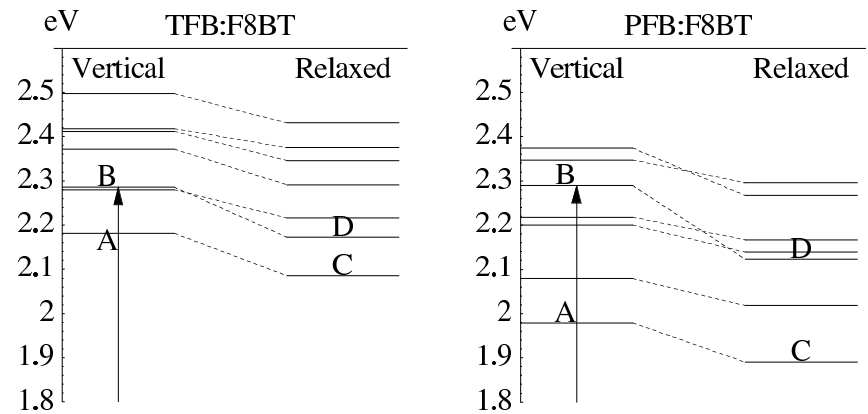

FIG. 5: Vertical and relaxed energies of the lowest lying states in the TFB:F8BT and PFB:F8BT heterojunctions. In each, $\mathrm{A}$ and $\mathrm{C}$ refer to the interchain exciplex state and $\mathrm{B}$ and $\mathrm{D}$ refer to the predominantly intrachain F8BT exciton state.

In TFB:F8BT, the band off-set is less than the exciton binding energy and these materials exhibit excellent LED performance. On the other hand, devices fabricated from PFB:F8BT where the exciton binding energy is less than the off-set, are very poor LEDs but hold considerable promise for photovoltic devices. In both of these systems, the lowest energy state is assumed to be an interchain exciplex as evidenced by a red-shifted emission about 50 80ns after the initial photoexcitation. 24] In the case of TFB:F8BT, the shift is reported to be $140 \pm 20 \mathrm{meV}$ and in PFB:F8BT the shift is $360 \pm 30 \mathrm{meV}$ relative to the exciton emission, which originates from the F8BT phase. Bearing this in mind, we systematically varied the separation distance between the cofacial chains from $r=2 a-5 a$ (where $a=$ unit lattice constant) and set $t_{\perp}=$ 0.01 in order to tune the Coulomb and exchange coupling between the chains and calibrate our parameterization.

For large interchain separations, the exciton remains localized on the F8BT chain in both cases. As the chains come into contact, dipole-dipole and direct Coulomb couplings become significant and we begin to see the effect of exciton destabilization. For TFB:F8BT, we select and interchain separation of $r=2.8 a$ giving a $104 \mathrm{meV}$ splitting between the vertical exciton and the vertical exciplex and $87.4 \mathrm{meV}$ for the adiabatic states. For PFB:F8BT, we chose $r=3 a$ giving a vertical exciton-exciplex gap of $310 \mathrm{meV}$ and an adiabatic gap of $233 \mathrm{meV}$. In both TFB:F8BT and PFB:F8BT, the separation produce interchain exciplex states as the lowest excitations. with energies reasonably close to the experimental shifts.

Fig. IV]compares the vertical and adiabatic energy levels in the TFB:F8BT and PFB:F8BT chains and Figs. 6 and 7 show the vertical and relaxed exciton and chargeseparated states for the two systems. Here, sites 1-10 correspond to the TFB or PFB chains and 11-20 correspond to the F8BT chain. The energy levels labeled in Fig. IV] correspond to the states plotted in Figs. [6 and 7 We shall refer to states $\mathrm{A}$ and $\mathrm{B}$ as the vertical exciplex and vertical exciton and to states C and D as the adiabatic exciplex and adiabatic exciton respectively. Roughly, speaking a pure exciplex state will have the 

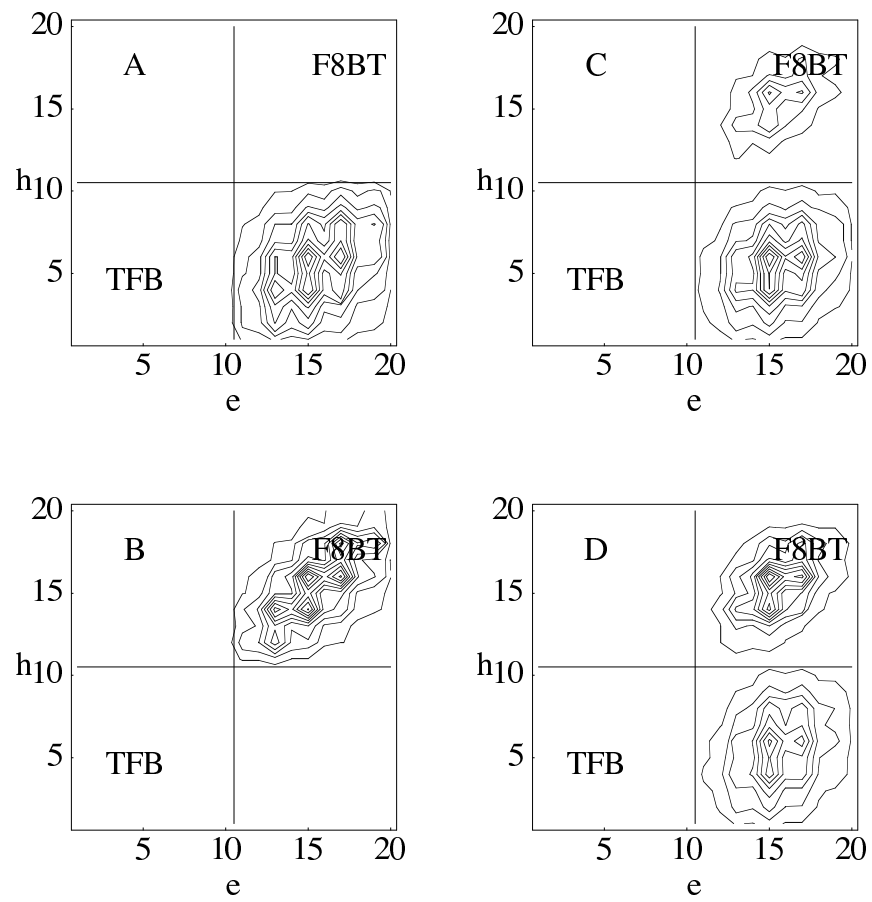

FIG. 6: Excited state electron/hole densities for TFB:F8BT heterojunction. The electron/hole coordinate axes are such that sites 1-10 correspond to TFB sites and 11-20 correspond to F8BT sites. Note the weak mixing between the interchain charge-separated states and the F8BT exciton in each of these plots.

charges completely separated between the chains and will contain no geminate electron/hole configurations. Likewise, strictly speaking, a pure excitonic state will be localized to a single chain and have only geminate electron/hole configurations. Since site energies for the the F8BT chain are modulated to reflect to internal chargeseparation in the F8BT co-polymer as discussed above, we take our "exciton" to be the lowest energy state that is localized predominantly along the diagonal in the F8BT "quadrant".

In the TFB:F8BT junction, the lowest excited state is the exciplex for both the vertical and adiabatic lattice configurations with the hole on the TFB and the electron on the F8BT. (Fig 6] A,C) In the vertical case, there appears to be very little coupling between intrachain and interchain configurations. However, in the adiabatic cases there is considerable mixing between intraand inter-chain configurations. First, this gives the adiabatic exciplex an increased transition dipole moment to the ground state. Secondly, the fact that the adiabatic exciton and exciplex states are only $87 \mathrm{meV}$ apart means that at $300 \mathrm{~K}$, about $4 \%$ of the total excited state population will be in the adiabatic exciton.

For the PFB:F8BT heterojunction, the band off-set is greater than the exciton binding energy and sits squarely on the other side of the stabilization threshold. Here the lowest energy excited state (Figs. 7A and B) is the inter-
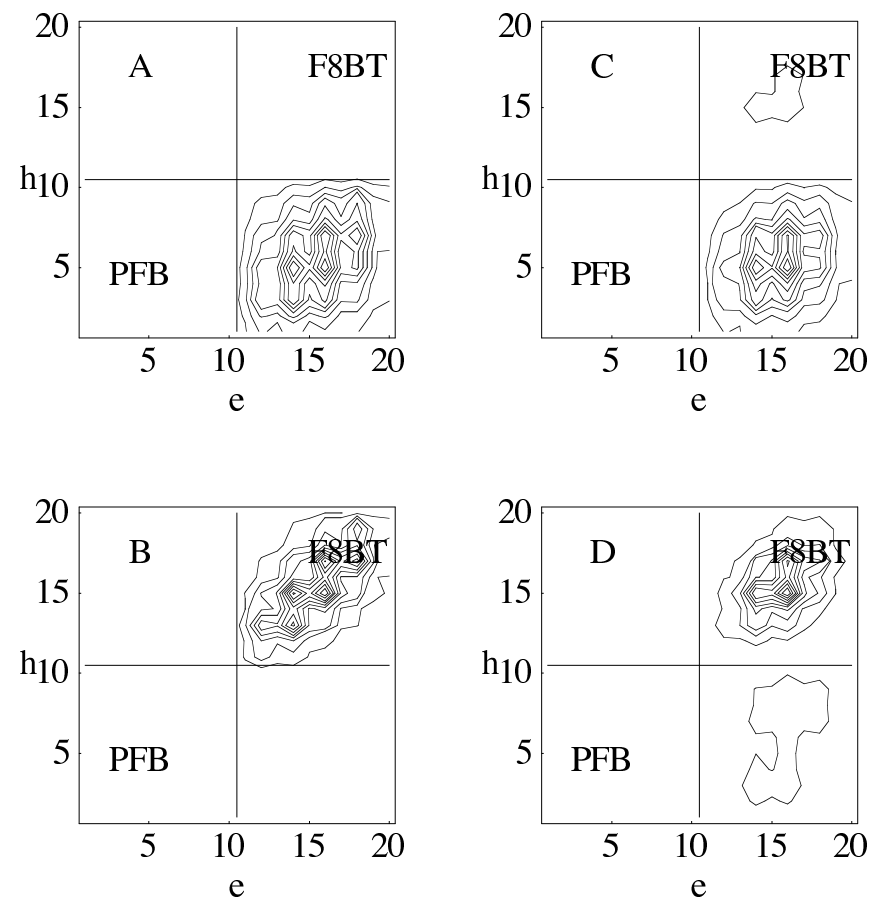

FIG. 7: Excited state electron/hole densities for PFB:F8BT heterojunction. The axes are as in previous figures except that sites 1-10 correspond to PFB sites and sites 11-20 to F8BT sites.

chain charge-separated state with the electron residing on the F8BT (sites 11-20 in the density plots in Fig (7) and the hole on the PFB (sites 1-10). The lowest energy exciton is almost identical to the exciton in the TFB:F8BT case. Remarkably, the relaxed exciton (Fig. 17D) shows slightly more interchain charge-transfer character than the vertical exciton (Fig. $7 \mathrm{C}$ ). While the system readily absorbs at $2.3 \mathrm{eV}$ creating a localized exciton on the F8BT, luminescence is entirely quenched since all population within the excited states is readily transfer to the lower-lying interchain charge-separated states with vanishing transition moments to the ground state.

In calculating the state-to-state interconversion rates for TFB:F8BT, we note two major differences between the static and the adiabatic Marcus-Hush approaches (See Fig. 8). First is the sparsity of the latter with transitions being limited to states with smaller energy differences. This leads to a relaxation dynamics that is more intertwined with the DOS. Second is the relatively faster rates calculated in the latter leading to interconversion lifetimes in the femtosecond (fs) to a couple of picosecond (ps) regime as opposed to hundreds of ps in the former. The same general difference is observed for PFB:F8BT (Not shown). These marked differences in the distribution of rates and their range of magnitudes are brought about by the introduction of the reorganization energy as a parameter in the rates calculation to complement the energy differences between the states. It provides a way to incorporate lattice distortions in the semiclassi- 


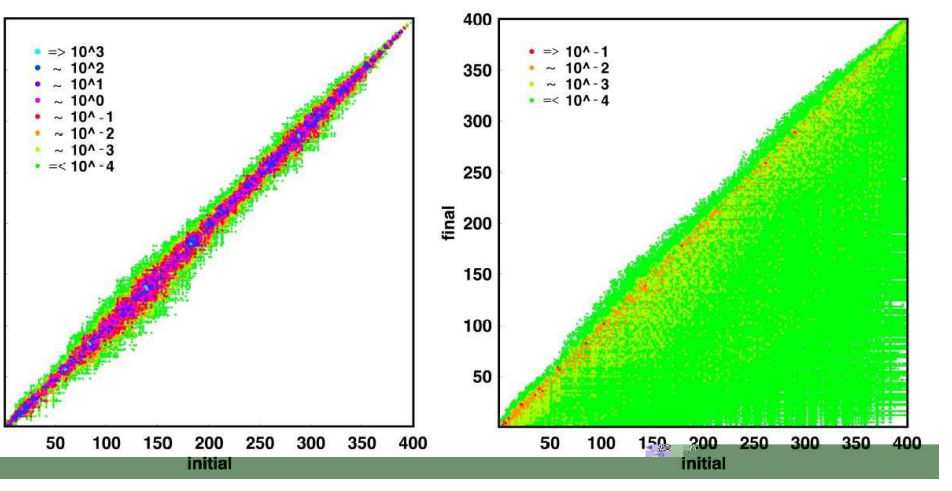

FIG. 8: (Color online) TFB-F8BT internal conversion rates distribution at $290 \mathrm{~K}$. Rates are in $\mathrm{ps}^{-1}$. Note the sparsity and relatively faster Marcus rates compared to the diabatic rates.

cal limit into the relaxation dynamics. While this is not fully dynamical in its account of the lattice distortions, it improves upon the static approximation previously employed.

The photoexcitation of heterojunction systems is simulated by populating a higher-lying excitonic state. Fig. 9 shows the time-evolved populations of the lowest chargetransfer (CT) and excitonic (XT) vertical and relaxed states, respectively, in photoexcited TFB:F8BT and PFB:F8BT. We see, in both approaches, that the relaxation to the lowest CT state is faster in TFB:F8BT than in PFB:F8BT. Furthermore, the relaxation from the XT state to the CT state occurs faster in the former. This is despite the XT state being formed faster in the latter for both cases. This is manifested more in the Marcus-Hush approach shown in Fig. 9 where despite reaching a maximum population of 0.86 in $250 \mathrm{fs}$ as opposed to just 0.40 in $500 \mathrm{fs}$, the $\mathrm{XT} \rightarrow \mathrm{CT}$ interconversion is practically done in 2 ps in TFB:F8BT compared to 10 ps in PFB:F8BT. In addition, we note that the $\mathrm{XT}$ state reaches a steady state population in TFB:F8BT whereas it goes to zero in PFB:F8BT. This small but non-zero population of the $\mathrm{XT}$ state is consistent with the distributed thermal population of states of 0.022 at $290 \mathrm{~K}$ owing to the fact that this $\mathrm{XT}$ state is $95 \mathrm{meV}$ higher in energy relative to the lowest CT state. [18]

Interestingly, while the overall $\mathrm{XT} \rightarrow \mathrm{CT}$ interconversion occurs in just a couple of ps in both heterojunction systems, a closer look into the rates reveal that this relaxation does not occur directly. Rather, it involves the next lowest CT state. Fig. 10 show the relevant interconversions between the three lowest states of both systems: the lowest CT state(CT1), the next lowest CT state(CT2), and the lowest XT state(XT). It is worth noting that the

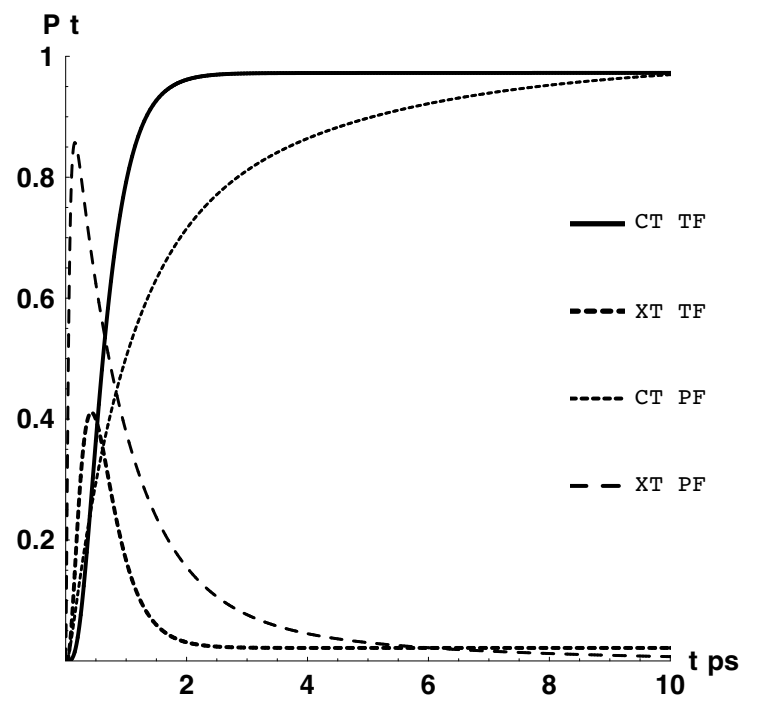

FIG. 9: Time-evolved populations of the lowest CT (solid lines) and XT (dashed lines) relaxed states of TFB:F8BT(TF) and PFB:F8BT(PF) in the Marcus-Hush approach at $290 \mathrm{~K}$.

considerable mixing between the intra-chain and interchain configurations of the former compared to those of the latter. In TFB:F8BT, the direct $\mathrm{XT} \rightarrow \mathrm{CT} 1$ transition $\left(\sim 10^{-3} \mathrm{ps}^{-1}\right)$ is at least 3 orders of magnitude slower than the corresponding $\mathrm{XT} \rightarrow \mathrm{CT} 2 \rightarrow \mathrm{CT} 1$ transition route $\left(>1 \mathrm{ps}^{-1}\right)$, the indirect route being consistent with the evolution data (Fig. 9). Thus, the $\mathrm{XT} \rightarrow \mathrm{CT}$ conversion occurs via the CT2 state and not directly. The reverse transitions for both routes are slower but have the same order of magnitude difference. This $\mathrm{CT} 1 \rightarrow \mathrm{CT} 2 \rightarrow \mathrm{XT}$ transition $\left(\sim 10^{-1} \mathrm{ps}^{-1}\right)$ effectively presents a regeneration pathway for the XT. This leads to an XT state population that is always at equilibrium with the CT1 state.

In PFB:F8BT the $\mathrm{XT} \rightarrow \mathrm{CT} 1$ and $\mathrm{XT} \rightarrow \mathrm{CT} 2$ conversion occur at relatively the same rate $\left(\sim 10^{-1} \mathrm{ps}^{-1}\right)$ while their reverse transitions are at least 2 orders of magnitude slower. Consequently, XT is not regenerated. The role played by CT2 as a bridge state is apparently relative to whether it has a slightly higher or lower energy than the XT as has been accounted by Morteani et al. 24, 25]. Spontaneous transition rates are typically faster when going from a higher to a lower energy state than the reverse according to detailed balance. Here, CT1 is the exciplex state which exhibit sizable mixing with the bulk CT state (CT2). When CT2 has a higher energy than $\mathrm{XT}$, such as in TFB:F8BT, a fraction of the population in $\mathrm{CT} 2$ converts to XT. If it has a lower energy relative to the $\mathrm{XT}$ state such as in PFB:F8BT, this regeneration of the XT, practically, does not occur.

To see the effect of temperature, the interconversion rates were calculated at 230, 290, and $340 \mathrm{~K}$. Fig. 11 shows how the interconversions among the three lowest 


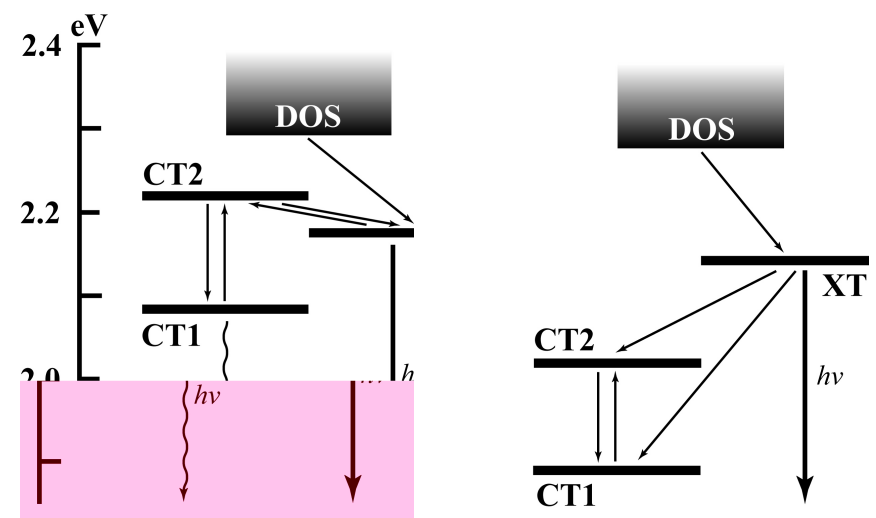

FIG. 10: Relevant Marcus-Hush interconversion rates for the 3 lowest states of (left) TFB:F8BT and (right) PFB:F8BT. In both cases, relaxation proceeds from the density of states (DOS) to the lowest excitonic state(XT) (off-set to the right relative to the CT states for clarity of relaxation route) before relaxing to the lowest charge-transfer state(CT1). CT1 proceeds to equilibrium with the next higher CT state(CT2). In PFB:F8BT, CT2 has a lower energy than XT where as in TFB:F8BT, it has a higher energy. Also shown are the radiative rates emanating from the $\mathrm{XT}$ states which are strongly coupled to ground state, $\mathrm{S}_{0}$, of both systems and the TFB:F8BT CT1 state which is just weakly coupled to $\mathrm{S}_{0}$.

states (CT1, CT2, and XT) of TFB:F8BT, as illustrated in Fig. [10] vary with temperature. This dependence is given in an Arrhenius plot of $\log \mathrm{k}$ vs $1 / \mathrm{T}$ and gives a linear plot for each transition having a slope associated with the activation energy, $E_{a c t}$, for that particular transition. This activation energy has the expression,

$$
E_{a c t}=\frac{\left(\Delta E-E_{\lambda}\right)^{2}}{4 E_{\lambda}} .
$$

Transitions to lower energy states are given as solid lines while those going to higher energy states are given as dashed lines. Curiously, although $\mathrm{XT} \rightarrow \mathrm{CT} 1$ is exothermic compared to $\mathrm{XT} \rightarrow \mathrm{CT} 2$ which is endothermic, the latter is a more favorable transition. This has to do with the fact that $\mathrm{XT} \rightarrow \mathrm{CT} 1$ has an activation energy almost three times greater than that of $\mathrm{XT} \rightarrow \mathrm{CT} 2$. As alluded to above, this is a consequence of the former being in the inverted region while the latter being in the normal region. In the inverted region, the larger $\Delta E$ is, the larger $E_{a c t}$ as opposed to the more familiar normal region where $E_{a c t}$ decreases as $\Delta E$ increases. Having stated this, however, we note that in the former, due to maximal overlap between the vibrational modes of the two states, transitions may be possible via tunneling processes. Overall, in TFB:F8BT, we see an increase in the fraction of the total excited state population in XT as temperature increases. At $230 \mathrm{~K}$ only $0.81 \%$ is in XT while at 290 and $340 \mathrm{~K}, 2.16 \%$ and $3.67 \%$ is in XT, respectively.

Finally, we note that all transition rates increase with temperature except for the CT2 $\rightarrow \mathrm{XT}$ in TFB:F8BT and $\mathrm{XT} \rightarrow \mathrm{CT} 2$ in PFB:F8BT which decrease with temperature. Such a trend, while not uncommon in chemical reactions, are though to be indicative of a more complicated transition mechanism as noted by Porter [26]. We surmise this to be due to the coupling between the low frequency vibrational modes of the initial state with the

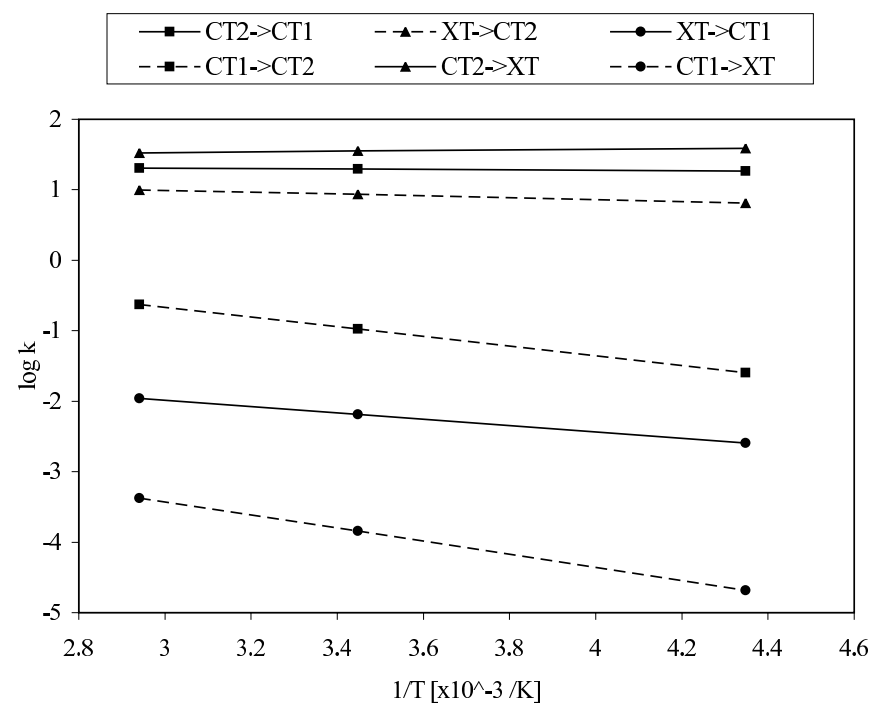

FIG. 11: Interconversion rates between the 3 lowest states of TFB:F8BT as a function of temperature (230, 290, and $340 \mathrm{~K})$. Plot is given as $\log \mathrm{k}$ vs $1 / \mathrm{T}$. Transitions to lower energy states are given as solid lines while their reverse are given as dashed lines. The CT2 $\leftrightarrow \mathrm{CT} 1, \mathrm{CT} 2 \leftrightarrow \mathrm{XT}$ and XT $\leftrightarrow \mathrm{CT} 1$ are plotted as squares, triangles and circles, respectively. All transition rates increase directly with temperature except the CT2 $\rightarrow$ XT conversion which decreases as temperature increases.

high frequency vibrational modes of the final state as in the case of an early transition state in reactive scattering. What we are definite of is that both transitions are exothermic and in the normal region which makes one speculate if there is any connection.

\section{DISCUSSION}

In this paper, we gave an overview of our recent work in developing a theoretical understanding interfacial excitonic dynamics in a complex material system. The results herein corroborate well with the experimental results on these systems. In particular, following either charge injection or photo-excitation, the system rapidly relaxes to form the interchain charge-separated species. In the experimental data, this occurs within the first 10 or so ps for the bulk material. Our calculations of a single pair of cofacial chains puts the exciplex formation at about 1 ps. Likewise, the experimental time-resolved emission indicates the regeneration occurs on a much longer timescale with most of the time-integrated emission coming from regenerated excitons. This too, is shown in our calculations as evidenced by the slow thermal repopulation of the XT state in the TFB:F8BT system. Since this state has a significant transition dipole to the ground state, population transfered to this state can either decay back to the CT state via thermal fluctuations or decay to the ground state via the emission of a photon. Since this secondary emission is dependent upon the thermal 
population of the XT state at any given time, the efficiency of this process shows a strong dependency upon the temperature of the system.

The exciton model we present herein certainly lacks the molecular level of details so desired by materials chemists. However, it offers a tractable way of building from molecular considerations the salient physical interactions that give rise to the dynamics in the excited states of these extended systems. In building this model we make a number of key assumptions. First, and perhaps foremost, that the excited states are well described via bands of $\pi$ orbitals and that from these bands we can construct localized Wannier functions. Hand in hand with this assumption is that within the general class of oligo-phenylene derived polymers, configuration interaction matrix elements, hopping integrals, electron/phonon couplings, and phonon spectra are transferable from one system to another. This is a fairly dangerous approximation since it discounts important contributions from heteroatoms, side-chains, and chain morphology. However, given that a single oligomeric chain of F8BT with 10 repeat units has well over 300 atoms, such potentially dire approximations are necessary in order to extract the important features of these very extended systems.

Secondly, we make the assumption that the explicit vibrational dynamics can be integrated out of the equations of motion for the electronic states. This is probably not too extreme of an assumption so long as we can assume that the phonons remain thermalized over the course of the electronic relaxation. However, looking back at the level correlation diagrams, crossings between diabatic states are present in this system and hence conical intersections between electronic states may play an important role. Finally, we discount the effects of electronic coherence. This, too, may have a profound impact upon the final state-to-state rate constants since it is well recognized that even a small amount of quantum coherence between states leads to a dramatic increase in the transition rate. Fortunately, many of the papers presented in this proceedings address these assumptions. Approaches, such as the MCTDH method presented by Thoss, the DFT based non-adiabatic molecular dynamics approach (NAMD) developed by Prezhdo, for example, are important strides towards achieving a molecular level understanding of complex photophysical processes in light-emitting and light-harvesting materials.

\section{ACKNOWLEDGMENTS}

This work was sponsored in part by the National Science Foundation and by the Robert A. Welch Foundation. The authors also thank the organizers for putting together a highly stimulating conference in a wonderful location.
[1] R.H.Friend, R.W.Gymer, A.B.Holmes, J.H.Burroughes, R.N.Marks, C.Taliani, D.D.C.Bradley, D.A. dosSantos, J.L.Bredas, M.Lögdlund, and W.R.Salaneck. Electroluminescence in conjugated polymers. Nature, 397:121128, 1999.

[2] M. Wohlgenannt, X. M. Jiang, Z. V. Vardeny, and R. A. J. Janssen. Conjugation-length dependence of spin-dependent exciton formation rates in pi -conjugated oligomers and polymers. Physical Review Letters, 88(19):197401, 2002.

[3] David Beljonne, Zhigang Shuai, AiJun Ye, and Jean-Luc Bredas. Charge-recombination processes in oligomer- and polymer-based light-emitting diodes: A molecular picture. Journal of the Society for Information Display, 13(5):419-427, 2005.

[4] A. Ye, Z. Shuai, and J. L. Bredas. Coupled-cluster approach for studying the singlet and triplet exciton formation rates in conjugated polymer led's. Physical Review B (Condensed Matter and Materials Physics), 65(4):045208, 2002.

[5] Z. Shuai, D. Beljonne, R. J. Silbey, and J. L. Bredas. Singlet and triplet exciton formation rates in conjugated polymer light-emitting diodes. Physical Review Letters, 84(1):131-134, 2000.

[6] M. Wohlgenannt, Kunj Tandon, S. Mazumdar, S. Ramasesha, and Z. V. Vardeny. correction: Formation cross-sections of singlet and triplet excitons in piconjugated polymers. Nature, 411(6837):617-617, 2001.

[7] Mark N. Kobrak and Eric R. Bittner. Quantum molec- ular dynamics study of polaron recombination in conjugated polymers. Physical Review B (Condensed Matter and Materials Physics), 62(17):11473-11486, 2000.

[8] Mark N. Kobrak and Eric R. Bittner. A dynamic model for exciton self-trapping in conjugated polymers. i. theory. The Journal of Chemical Physics, 112(12):53995409, 2000.

[9] Mark N. Kobrak and Eric R. Bittner. A quantum molecular dynamics study of exciton self-trapping in conjugated polymers: Temperature dependence and spectroscopy. The Journal of Chemical Physics, 112(17):7684-7692, 2000.

[10] Alexander L. Burin and Mark A. Ratner. Spin effects on the luminescence yield of organic light emitting diodes. The Journal of Chemical Physics, 109(14):6092-6102, 1998.

[11] Kunj Tandon, S. Ramasesha, and S. Mazumdar. Electron correlation effects in electron-hole recombination in organic light-emitting diodes. Physical Review B (Condensed Matter and Materials Physics), 67(4):045109, 2003.

[12] M. Wohlgenannt, C. Yang, and Z. V. Vardeny. Spindependent delayed luminescence from nongeminate pairs of polarons in pi-conjugated polymers. Physical Review $B$ (Condensed Matter and Materials Physics), 66(24):241201, 2002.

[13] M. Wohlgenannt and O. Mermer. Single-step multiphonon emission model of spin-dependent exciton formation in organic semiconductors. Physical Review B (Con- 
densed Matter and Materials Physics), 71(16):165111, 2005.

[14] William Barford. Theory of singlet exciton yield in lightemitting polymers. Physical Review B (Condensed Matter and Materials Physics), 70(20):205204, 2004.

[15] Stoyan Karabunarliev and Eric R. Bittner. Dissipative dynamics of spin-dependent electron-hole capture in conjugated polymers. The Journal of Chemical Physics, 119(7):3988-3995, 2003.

[16] Stoyan Karabunarliev and Eric R. Bittner. Spindependent electron-hole capture kinetics in luminescent conjugated polymers. Physical Review Letters, 90(5):057402, 2003.

[17] Eric R. Bittner and Stoyan Karabunarliev. Energy relaxation dynamics and universal scaling laws in organic light-emitting diodes. International Journal of Quantum Chemistry, 95(4-5):521-531, 2003.

[18] Eric R. Bittner, John Glenn Santos Ramon, and Stoyan Karabunarliev. Exciton dissociation dynamics in model donor-acceptor polymer heterojunctions. i. energetics and spectra. The Journal of Chemical Physics, 122(21):214719, 2005.

[19] John Glenn Santos Ramon and Eric R. Bittner. Exciton dissociation dynamics in model donor-acceptor polymer heterojunctions. ii. kinetics and photophysical pathways. The Journal of Chemical Physics-submitted, 2005.

[20] Stoyan Karabunarliev and Eric R. Bittner. Polaron- excitons and electron-vibrational band shapes in conjugated polymers. The Journal of Chemical Physics, 118(9):4291-4296, 2003.

[21] Stoyan Karabunarliev and Eric R. Bittner. Electroluminescence yield in donor-acceptor copolymers and diblock polymers: A comparative theoretical study. The Journal of Chemical Physics, 108(29):10219-10225, 2004.

[22] P. Vogl and D. K. Campbell. First-principles calculations of the three-dimensional structure and intrinsic defects in trans-polyacetylene. Physical Review B (Condensed Matter), 41(18):12797-12817, 1990.

[23] Z G Yu, M W Wu, X S Rao, X Sun, and A R Bishop. Excitons in two coupled conjugated polymer chains. Journal of Physics: Condensed Matter, 8(45):8847-8857, 1996.

[24] Arne C. Morteani, Anoop S. Dhoot, Ji-Seon Kim, Carlos Silva, Neil C. Greenham, Craig Murphy, Ellen Moons, Salvatore Ciná, Jeremy H. Burroughes, and Richard H. Friend. Barrier-free electron-hole capture in polymer blend heterojunction light-emitting diodes. Advanced Materials, 15(20):1708, 2003.

[25] Arne C. Morteani, Paiboon Sreearunothai, Laura M. Herz, Richard H. Friend, and Carlos Silva. Exciton regeneration at polymeric semiconductor heterojunctions. Physical Review Letters, 92(24):247402, 2004.

[26] George Porter. Flash photolysis and some of its applications. Nobel Lecture, pages 241-263, 1967. 